\title{
Battery Energy Storage Cost and Capacity Optimization for University Research Center
}

\author{
Mojtaba Moghimi, Rasoul Garmabdari, Sascha Stegen, and Junwei Lu
}

\begin{abstract}
Microgrids (MGs) are the essential part of the modern power grids defined as the building blocks of smart grids. Renewable Energy Sources (RESs) and Battery Energy Storage Systems (BESSs) combined with Distributed Generators (DGs) form a comprehensive MG, which require the control and Energy Management System (EMS) to fulfill the load and grid requirements. As the need for BESS grows due to uncertainties of RESs, scheduling and cost management of BESSs in the MG becomes more of a concern. In this paper, BESSs have been designed for a university research center to simultaneously overcome the outage problem and shave the peak demand considering the BESS sizing and degradation; MG cost minimization, as well as MG scheduling. PV and wind are the RESs employed in this study and in combination; Li-Ion BESS has been utilized to investigate the MG performance. A two-layer optimization algorithm has been presented to optimally define the BESS size and minimize the operational cost of the MG achieving the peak shaving and valley filling objectives. The results prove the functionality and applicability of the proposed system to be implemented as a part of the experimental MG at Griffith University in order to enhance the stability and reliability of the research center and at the same time minimize the operational costs of the MG.
\end{abstract}

Index Terms-MG, Energy Management System, Battery Sizing, Cost Minimization, Peak Shaving and Valley Filling, Power fluctuations, Battery Degradation.

\section{INTRODUCTION}

$\mathrm{W}$ ITH the increasing penetration of renewables into the power grid, MGs are necessary to overcome the new challenges. MGs consisting of Renewable Energy Sources (RESs), Distributed Generators (DGs), and the Battery Energy Storage Systems (BESSs), can provide the solution for the power grid modernization. Nowadays, RESs such as Photovoltaics (PV) and Wind Turbines (WT) are frequently used as the clean energy resources in the power applications reducing the $\mathrm{CO}_{2}$ emission [1]. Although RESs offer a lot of benefits to the grid but at the same time bring up new challenges. Due to the uncertainty and intermittency of PV and WT in the MGs, employing BESSs along with the RESs is a solution to enhance the reliability of MG as well as the capability to maximize its benefits by scheduling and managing the energy.

University research centers, modeled as MGs, require stable and continuous power to run experiments and thus sometimes sensitive equipment. Implementing the MG concept to the research center enables the research center to function as an Uninterruptable Power Supply (UPS). One of the primary issues to deal with in the research center is power fluctuation. Power peaks and power drops can damage the equipment, cause power inefficiencies, and consequently can have a negative impact on the quality of research. As of today, employing BESSs as a part of MG to shave the peak and fill the valley in the demand curve during normal research center operation is the best solution to overcome the aforementioned problems. However, the appropriate capacity of the employed BESS, its constraints, investment and operation costs, and degradation are the important parameters, which need to be considered. In order to achieve this, a novel BESS scheduling and sizing optimization algorithm is developed.

To implement the BESS in the research center MG optimally, fulfilling two major objectives is crucial. The first objective is finding the optimum BESS capacity and the second one is minimizing the cost of the MG management with the defined BESS capacity. To achieve the cost objective, a scheduling algorithm to minimize the cost is required, which optimizes the battery charge/discharge time considering RESs operation, BESS constraints, peak power and power drop charges, and MG operational costs. On the other hand, to fulfill the capacity objective, an optimization algorithm should be employed to determine the required capacity of the BESS, which offers the highest benefit to the research center MG.

Previous studies in the literature have developed different approaches to optimize the BESS scheduling and sizing. Most of the papers in the literature have focused on either sizing or scheduling and have considered BESS sizing and scheduling as one model. In addition, there are other major factors, which have significant impact on BESS sizing and MG cost optimization. BESS aging, peak shaving and valley filling are the key ones. Authors in researches [2, 3] have investigated sizing of BESSs for MGs including RESs such as PV and wind whereas no cost minimization of MG or BESS scheduling have been taken into account in these studies. Furthermore, paper [4] has focused on BESS sizing investigating peak demand shaving by employing BESS but no scheduling of BESS is measured. Some previous studies inspect the MG cost minimization and peak demand shaving but have not examined BESS sizing in their optimization model [5-8]. In addition, scheduling systems for BESS have been established in other projects to reduce the peak demand utilizing the RESs such as PV. However, sizing of the BESS is missing in these studies $[9,10]$. 
Further research projects investigated sizing and scheduling of the BESS to minimize the MG costs. One of the important factors that impacts total operation cost of MGs is peak demand charges. Therefore, the more the peak demand is reduced, the lower the costs of the MG become. Researchers in [1, 11-14] have developed a sizing and operation optimization strategy to primarily minimize the costs of the MG and shave the peak demand employing the BESS in combination with RESs (PV). Nevertheless, none of these studies has measured the BESS degradation as an influential element in their models.

BESS aging is a major factor affecting the total operational and maintenance cost of the MG. In order to prolong the life of the BESS, the cycle aging and calendar aging process of the BESS should be reviewed in the scheduling and cost minimization algorithm of MGs [28]. Papers [15-17] inspect the sizing and scheduling of BESS employing different optimization methods to minimize the costs evaluating peak demand shaving and BESS degradation. In these studies, PV and wind as the main RESs play their role in the scheduling procedure of MG combined with DGs in some of the cases.

Different optimization approaches are examined in earlier literature to solve the MG cost minimization problem. Sizing and scheduling of BESSs in the MG are explored to minimize the costs applying dynamic programing [11], [18]. The drawback of dynamic programming is the complexity in case of large systems. Linear programming as a simpler optimization method has been employed in the researches $[8]$ and $[19,20]$ to manage the energy of RESs, DGs, BESSs and loads in the MG and reduce the operational and maintenance costs respectively. Authors in $[21,22]$ have conducted a research on BESS sizing and cost minimization employing Genetic Algorithm (GA) in order to optimize the system. The drawback of the GA is its uncertainty in the results. To improve the accuracy of the achieved results, papers [23, 24] have utilized Particle Swarm Optimization (PSO) method to minimize the costs and attain the best BESS size. Furthermore, researchers in $[25,26]$ have implemented a two-layer optimization approach to minimize the costs of MG utilizing PSO algorithm.

In this paper, a two-layer optimization algorithm is developed to find the optimal size of the BESS for the university research center while minimizing the operational costs of the MG and power drops. The inner optimization layer minimizes the costs related to the BESS and research center operations such as peaks and outages modeling as a Mixed Integer Quadratic Programming (MIQP) while the outer optimization layer defines the best BESS capacity based on the results of the inner layer. The cost analysis is executed to validate the performance of the optimization algorithm. The rest of the paper is as follows. Section II describes the system configuration and modelling of the problem. In Section III, BESS optimization algorithm, sizing and cost minimization have been explained. Results and analysis are discussed in section IV and the paper concludes with section V.

\section{II.SYSTEM CONFIGURATION}

The system under study at the university consists of an experimental MG and the research center. The experimental
MG at the University is a testing facility developed for the power grid testing purposes. The MG testing facility is a hybrid AC/DC system that includes a PV system with the power of 20 $\mathrm{kW}$ peak, $70 \mathrm{kWh}$ energy storage system, a $4 \mathrm{~kW} \mathrm{WT}$, which is not connected to the grid yet, and a DC bus connected to the research center. The research center includes a semiconductor technology facility established for Micro- and Nanotechnology research activities. It is equipped with sensitive and high accuracy devices that require stable and continuous electrical power supply. A $100 \mathrm{~kW}$ PV system is installed on the roof of the research center and a $10 \mathrm{~kW}$ WT is planned to install. The configuration of the system under study is illustrated in Fig. 1.

\section{A. University Research Center}

One of the main concerns in the research center is power fluctuations and outages happening during the experiments.

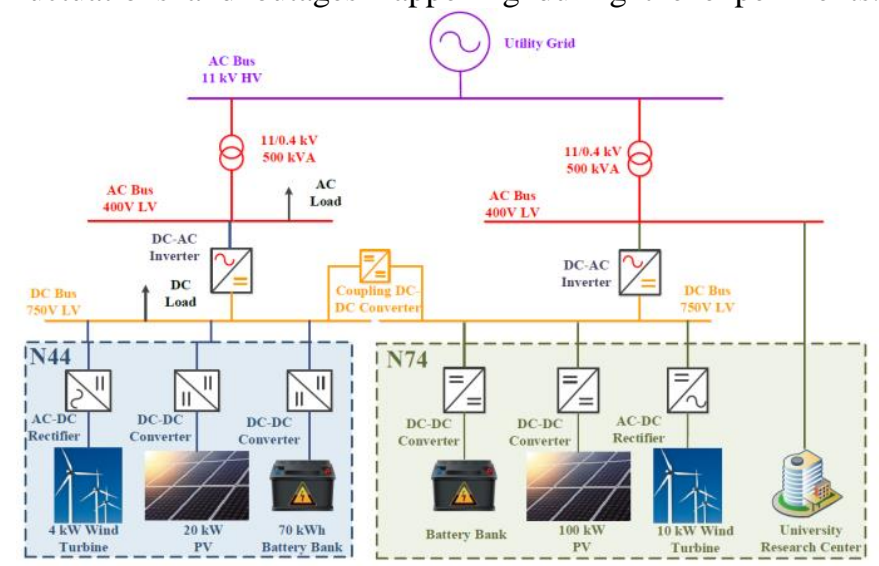

Fig. 1. Microgrids Configuration

Due to high sensitivity of the equipment in the center and high precision of the experiments, a non-interrupted continuous power to the loads is required. At some occasions such as bad weather conditions or poor power network, power outages happen in the research center lasting from several minutes to one day. On the other hand, large peaks are realized in the load profile of research center, which will impose extra costs on the research center.

\section{B. WT power}

WT and PV are the commonly used RESs in MGs. Currently, no WT has been installed at the university campus but a $4 \mathrm{~kW}$ and a $10 \mathrm{~kW}$ WT are planned to be installed for the experimental MG and research center, respectively. The important characteristics of WT are the rated power, $P_{W T_{-} r}$ and rated speed, $v_{r}$. The real data for wind speed, $v(t)$ for a duration of one year has been collected from the weather station located in the university campus for wind monitoring purposes. The output power of WT, $P_{W T}$ is described as in (1):

$$
P_{W T}=\left\{\begin{array}{lr}
0 & v(t)<v_{c i} \text { or } v(t)>v_{c o} \\
P_{W T_{-} r} \frac{v(t)-v_{c i}}{v_{r}-v_{c i}} & v_{r} \leq v(t) \leq v_{c i} \\
P_{W T_{-} r} & v_{r} \leq v(t) \leq v_{c o}
\end{array}\right.
$$

where $v_{c i}$ and $v_{c o}$ are the cut-in and cut-out wind speeds, respectively [27]. There is no cost related to WT considered in 
this study.

\section{Solar PV power}

The solar PV is another popular form of RESs employed to generate electrical power. For this study, two sets of PV modules have been employed. A $20 \mathrm{~kW}$ PV module for the experimental MG and a $100 \mathrm{~kW}$ PV module for the research center have been installed. PV modules are connected to the DC bus via the DC/DC converters. In order to apply PV characteristics to the optimization algorithm, real PV data is required. The PV data for one year has been collected from the MGs using the data acquisition and monitoring system with 1 min interval. The data acquisition system is employed to collect necessary information and monitor the performance of the MGs [28]. No operation and maintenance costs has been measured for PV.

\section{BESS}

One of the problems with RESs is their intermittency and uncertainty to generate power. Solar PV and WTs are dependent to the weather conditions such as sunny or cloudy and wind speed. Due to aforementioned problem, the RESs are not solely sufficient to operate as reliable generation source. Employing BESS alongside RESs is suggested as a solution to provide a more reliable source of generation. BESS are capable of charging and discharging based on the preplanned schedules. In other words, BESS charges at the times that energy is not needed and discharges when it is needed. On the other hand, to operate the BESS optimally, battery characteristics such as battery model, operation and maintenance cost, degradation, and capacity should be taken into account.

In this study, a $70 \mathrm{kWh}$ Li-Ion BESS has been installed for the experimental MG. Nonetheless, the research center has no operational BESS and BESS installation is planned for the near future. To model the BESS operation, charge and discharge mode of BESS should be considered. The energy of BESS, $E_{B E S S}(t)$ is described in (2):

$$
\begin{aligned}
& E_{B E S S}(t)=E_{B E S S}(t-\Delta T)+\left(\eta_{c h} P_{c h}(t-\Delta T)-\right. \\
& \left.\frac{P_{\text {disch }}(t-\Delta T)}{\eta_{\text {disch }}}\right) \cdot \Delta T
\end{aligned}
$$

In the energy equation of BESS, energy at the time $t$ is obtained based on the available energy at the previous time step and charge and discharge power rates of the BESS, $P_{c h}$ and $P_{\text {disch }}$ at time $t-\Delta T$ considering charge and discharge efficiencies, $\eta_{c h}$ and $\eta_{\text {disch }}$. In addition to the BESS energy equation, BESS is limited to operational constraints. The BESS operates in a predefined range of State of Charge (SOC).

$S O C_{\min } \leq S O C(t) \leq S O C_{\max }$

The other important factor in BESS modeling is degradation. The BESS works at different SOCs, which would have different impact on the BESS degradation. The BESS ages either due to calendar aging or cycle aging [29]. The cycle aging is primarily dependent on the Depth of Discharge (DOD) of BESS, where
DOD is defined as the energy in one charging or discharging event with respect to the full capacity. The battery lifetime, $L(D O D)$ with respect to DOD is formulated as in (4).

$L(D O D)=a \times D O D^{-b} \times \exp (-c \times D O D)$

where $a, b$, and $c$ are curve-fitting coefficients [30]. In addition, the operation of BESS imposes costs on the system, as the cost is mainly dependent on DOD. The BESS operation cost, $\mathrm{C}_{\mathrm{op}}$ (DOD) is described as follows [31]:

$$
\mathrm{C}_{\mathrm{op}}(\mathrm{DOD})=\frac{C_{c a p}}{2 \cdot L(D O D) \cdot E_{B E S S} \cdot D O D \cdot \eta_{c h} \cdot \eta_{\text {disch }}}
$$

where $C_{c a p}$ is BESS price. Applying (5) to a Li-Ion battery, the operational cost is calculated for a specific DOD. For instance, $C_{o p}(0.7)$ means the BESS cycles between $30 \%$ and $100 \%$ SOC. Thus, different operation costs are considered for charge and discharge with different DODs in each cycle.

\section{E. Electricity grid policy}

University campus as a large customer for the utility has high consumption of electrical energy. The utility charges the university based on Time of Use (TOU) tariffs in different categories such as retail energy usage charges and network charges. The university is charged for both energy usage in $\mathrm{kWh}$ on a daily basis and demand usage in $\mathrm{kVA}$ on a monthly basis. The charges for energy usage are applied depending on the time of the day, being peak hours or off-peak hours, where university pays higher prices to the utility during peak hours. The TOU tariffs for large customers in Queensland are illustrated in Table I.

According to Table I, the electricity costs to consider are the purchased energy cost, $\mathrm{C}_{\mathrm{p}}(t)$ and peak demand cost, $\mathrm{C}_{\text {peak }}$, where $C_{p}(t)$ includes off-peak and peak energy prices and $\mathrm{C}_{\text {peak }}$ includes Distribution Use of System (DUoS) and Transmission Use of System (TUoS) charges. By reducing the peak (i.e. peak shaving), the impact of peak on the research center power fluctuations and on total utility charges decreases.

TABLE I

TIME OF USE (TOU) TARIFFS FOR THE UNIVERSITY

\begin{tabular}{|l|l|l|}
\hline Charges & Unit Price & Unit \\
\hline Retail Energy Usage Charges & & \\
\hline QLD Off-peak & 10.68 & $\mathrm{c} / \mathrm{kWh}$ \\
\hline Network Charges & 5.8 & $\mathrm{c} / \mathrm{kWh}$ \\
\hline DUoS Demand & & \\
\hline TUoS Demand & 8.073 & $\$ / K V A / M o n t h$ \\
\hline
\end{tabular}

The other electricity cost parameter considered in this study is the outage power cost, $\mathrm{C}_{\text {out }}$. The research center is supposed to work $24 / 7$ and if a power outage happens, it can damage the equipment and impose extra costs on the research center. Therefore, the outage cost is to prevent power outages in the worst-case scenario and fill the valleys in normal conditions. 


\section{BATTERY ENERGY STORAGE OPTIMIZATION ALGORITHM}

The BESS plays an important role in the MG Energy Management System (EMS) by optimal scheduling of the charge/discharge rate and time. Scheduling of the BESS can affect the total cost of the MGs as it reduces the purchased energy from grid through management of peak/off-peak hours load consumption, demand charges through peak shaving. Furthermore, it decreases the operational costs via considering the degradation and DOD, and the outage costs via valley filling. The other influential cost factor is the BESS price, which can be minimized by properly sizing of the BESS. The key objective of optimization algorithm is to minimize the costs of the research center by providing a suitably sized BESS. To achieve this objective, the optimization algorithm is designed in two layers, cost minimization and sizing. In the inner optimization layer, which is cost minimization, the objective function considers all the costs of system and optimizes the operation of the BESS. On the other hand, the outer optimization layer optimizes the size of the BESS based on the investment and operational costs. After defining the minimized cost and the best size for the BESS, cost analysis is applied to the results to evaluate the Payback Period (PBP) of the BESS investment. The overall BESS optimization framework is presented in Fig. 2.

\section{A. Cost Minimization Algorithm (Inner Optimization Layer)}

The inner layer of the optimization algorithm is to minimize the operational cost of the Microgrid, which includes the BESS operating cost, the peak cost and the power outage cost. To solve the optimization problem, it is modeled as an objective

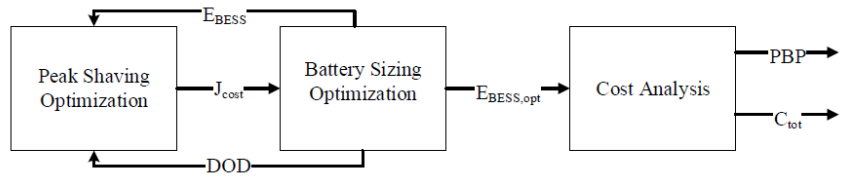

Fig. 2. BESS Optimization Framework

function with constraints.

\section{1) Objective Function}

The objective function contains all the cost parameters related to the BESS. It is modeled to schedule the charge and discharge operation of the BESS based on the TOU tariffs. In addition, the model undertakes the functions of shaving the peak, filling the valley, and minimizing the degradation of BESS within one operation period. The objective function is demonstrated as a mixed integer quadratic programming (MIQP) problem and is described as (6):

$J_{\text {cost }}=\sum_{t=t_{0}}^{T} C_{p}(t) \cdot P_{\text {grid }}(t) \cdot \Delta t+C_{o p, c h}(t) \cdot P_{B E S S, c h}(t) \cdot \Delta t+$ $C_{\text {op,disch }}(t) \cdot P_{B E S S, \text { disch }}(t) \cdot \Delta t+C_{\text {peak }} \cdot P_{\text {peak }}+C_{\text {out }} \cdot P_{\text {out }}$

where $P_{\text {grid }}(t), P_{B E S S, c h}(t), P_{B E S S \text {,disch }}(t), P_{\text {peak }}$, and $P_{\text {out }}$ are the purchase power from grid, the charged and discharged power of battery, the peak power of the month, and outage power. $C_{p}(t), C_{o p, c h}(t), C_{o p \text {,disch }}(t), C_{\text {peak }}$, and $C_{\text {out }}$ are the cost coefficient of the purchased energy from the grid, operating cost coefficient of the charge and discharging power of the battery, cost coefficient of the grid demand charges, and the power outage cost coefficient respectively. $t_{0}, T$ and $\Delta t$ represent the beginning, end and time of simulation period, respectively.

2) Constraints

The constraints, which are defined as a part of the optimization problem, determine the ranges in which the variables have to be operating. The first constraint is the network power balance between power generation and load consumption.

$P_{\text {grid }}(t)+P_{P V}(t)+P_{W T}(t)+P_{B E S S, \text { disch }}(t)-P_{B E S S, c h}(t)=$
$P_{\text {load }}(t)$

The next constraint is the BESS energy limits. The BESS should work between the maximum available energy, $E_{B E S S, \max }$, and the minimum available energy, $E_{B E S S, \min }$.

$E_{B E S S, \min } \leq E_{B E S S}(t) \leq E_{B E S S, \max }$

Furthermore, BESS has a limited charge and discharge power rate.

$0 \leq P_{\text {disch }}(t) \leq P_{\text {disch,max }}(t) \cdot \beta(t)$

$0 \leq P_{c h}(t) \leq P_{c h, \max }(t) \cdot(\beta(t)-1)$

where $\beta(t)$ is the binary variable to define charge/discharge mode of BESS. If $\beta(t)$ is equal to one, it means BESS is discharging, otherwise it is charging. The other constraint that should be taken into account is the initial and final energy of the BESS. The BESS operation starts from the same energy as it ended after one full operation period.

$E_{B E S S, \mathrm{t}_{0}}(t)=E_{B E S S, T}(t)$

The MIQP cost optimization problem is modeled in Matlab and IBM ILOG CPLEX MIQP optimization solver is employed to solve the problem.

\section{B. Battery Sizing Algorithm (Outer Optimization Layer)}

Larger capacity of the BESS enhances the investment costs, while higher BESS capacity does not necessarily mean lower operation costs. Therefore, there is a tradeoff between the operation costs and the investment costs. The sizing algorithm determines the optimal BESS capacity by finding the balancing point between various costs. The outer optimization layer deals with the determination of the appropriate size of the BESS considering the investment and operational costs of the BESS. The sizing algorithm gets the minimized cost of the system as input from the inner optimization layer and provides the optimal capacity of the BESS as output.

In order to minimize the costs in the sizing algorithm, it is required to calculate the total cost. Total cost, $C_{\text {tot }}$ consists of two parts: the cost of the BESS investment (i.e. capital cost per day, $\left.C_{\text {cap,pd }}\right)$ and the operational costs of the MGs as the result of inner cost minimization layer, $\mathrm{J}_{\text {cost }}$. In the calculation of $C_{\text {cap }, p d}$, the capacity of the BESS and the capital cost of the BESS, $C_{\text {cap }}$ are considered, where $C_{\text {cap }}$ is calculated based on 
the annual interest rate, $r_{i n}$ and the BESS lifetime, $L(D O D)$. The relationship between the parameters is formulated in (12) to (14).

$C_{t o t}=\mathrm{J}_{\mathrm{cost}}+C_{c a p, d}$

$C_{\text {cap }, d}=\frac{1}{365} \cdot C_{\text {cap }} \cdot E_{B E S S}$

$C_{c a p}=\frac{r_{i n \cdot\left(1+r_{i n}\right)^{L(D O D)}}}{\left(1+r_{i n}\right)^{L(D O D)}-1}$

By running the sizing optimization algorithm and minimizing the total cost for all the BESS capacities, the optimal size of the BESS, $E_{B E S S}^{o p t}$ is obtained. To solve the optimization problem, an iterative heuristic method is employed. The heuristic algorithms are effective methods to solve multi-dimensional optimization problems [32]. The BESS sizing optimization function is described as follows.

$\min _{X} F(X)=\left\{C_{t o t}\right\}$

where $X$ represents the decision variables of outer optimization layer and corresponds to the BESS capacity and DOD.

$X=\left\{E_{B E S S}, D O D\right\}$

After initializing the algorithm by the minimum values for the BESS capacity and DOD, at the $k$ th iteration of the sizing optimization algorithm, the total cost of the specific size and DOD is calculated. This process continues until the stop condition is met. The stop condition is satisfied in case that the optimal size of the BESS is achieved or the number of the iterations exceeds the maximum iteration limit. The BESS sizing optimization is demonstrated in Fig. 3.

\section{Cost-benefit Analysis}

Minimizing the costs and determining the BESS capacity are two important factors influencing the final cost analysis. In addition, the other factor that plays an important role is PBP.

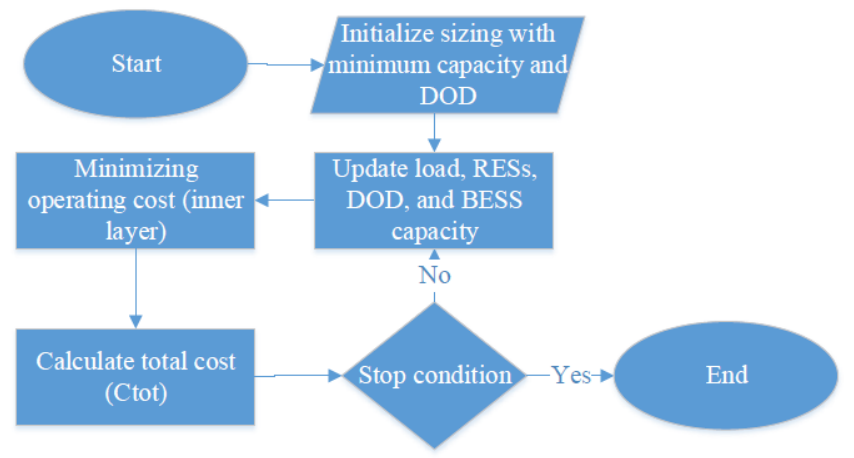

Fig. 3. BESS sizing optimization algorithm.

PBP indicates how long it takes to get profits from the investments with respect to the savings. To calculate the PBP, the annual savings are estimated and compared to that of the investments. Then it reaches to the point that savings and investments become equal. The formula is demonstrated in (17).

$\sum_{\mathrm{y}=1}^{P B P} S_{a n l} \cdot\left(1+r_{i n}\right)^{y-1} \geq C_{c a p}$

where $y$ is the index for the number of the years and $S_{a n l}$ is the savings per year considering the interest rate, $r_{i n} . S_{a n l}$ is evaluated as the decline occurring in costs due to the cost minimization algorithm.

\section{RESULTS AND ANALYSIS}

The performance of two-layer optimization system for different capacities of BESS in the MGs is investigated and analyzed. The minimum BESS capacity for the MGs is $70 \mathrm{kWh}$, which is specified to the experimental MG. The additional BESS capacity up to $800 \mathrm{kWh}$ in total is allocated to the research center to minimize the costs and reduce the power fluctuations. The results of the cost optimization and BESS sizing are investigated and discussed as follows. The parameters of the system under study are shown in Table II.

\section{A. Cost Optimization Analysis}

The cost optimization algorithm minimizes the operation costs of the research center considering the peaks and valleys.

TABLE II

PARAMETERS FOR THE CASE STUDY

\begin{tabular}{|l|l|l|}
\hline Parameters & Value & Unit \\
\hline $\boldsymbol{C}_{\boldsymbol{p}}(\boldsymbol{t})$ - Peak & 10.68 & $\mathrm{c} / \mathrm{kWh}$ \\
\hline $\boldsymbol{C}_{\boldsymbol{p}}(\boldsymbol{t})$ - Off-peak & 5.8 & $\mathrm{c} / \mathrm{kWh}$ \\
\hline $\boldsymbol{C}_{\text {peak }}$ & 9.3 & $\$ / \mathrm{KVA} /$ Month \\
\hline $\boldsymbol{C}_{\text {out }}$ & 2 & $\$ / \mathrm{KVA} /$ Month \\
\hline $\boldsymbol{C}_{\text {cap }}$ & 300 & $\$ / \mathrm{kWh}$ \\
\hline DOD & 80 & $\%$ \\
\hline $\boldsymbol{\eta}_{\boldsymbol{c h}}, \boldsymbol{\eta}_{\text {disch }}$ & 95 & $\%$ \\
\hline $\mathbf{L}$ & 10 & Years \\
\hline $\boldsymbol{r}_{\boldsymbol{i n}}$ & 10 & $\%$ \\
\hline
\end{tabular}

The peaks are shaved and the valleys are filled to reduce the costs and minimize the probability of the power outages. The peak day of a month in 2016 has been selected to investigate the performance of the cost optimization algorithm. The size of the BESS is chosen to be $280 \mathrm{kWh}$, and the scheduling runs for oneday operation. At the end of the daily cycle, the BESS is charged and discharged with different rates to meet the requirements of the scheduling system. The cost optimization result for one-day cycle is illustrated in Fig. 4.

As is shown in Fig. 4, the cost optimization algorithm reduces the peak of the day from $1362 \mathrm{~kW}$ to $1188 \mathrm{~kW}(174$ $\mathrm{kW}$ reduction) and fills the valley from $843 \mathrm{~kW}$ to $906 \mathrm{~kW}$ (63 $\mathrm{kW}$ growth). The BESS charges during the off-peak hours to increase the valleys and discharges during the peak hours to decrease the peak load of the day. 


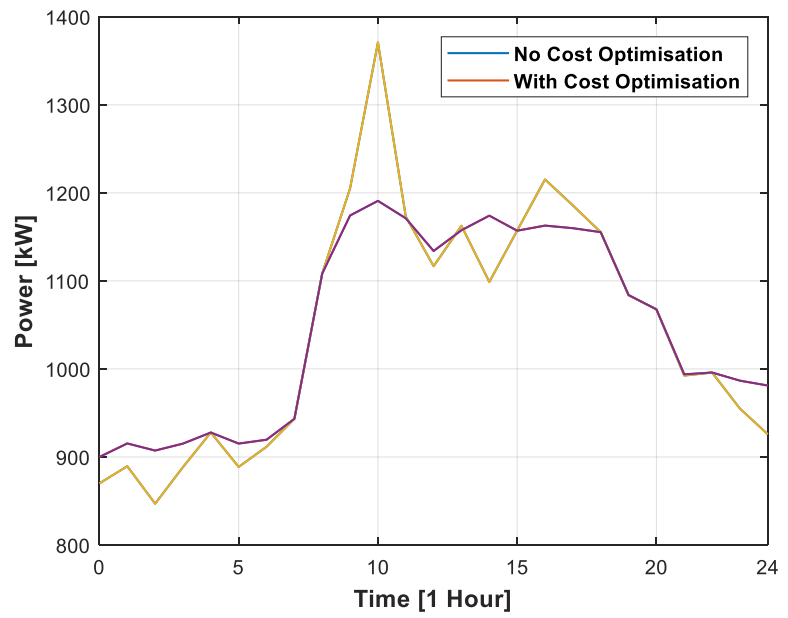

Fig. 4. Cost Optimization for a peak day of the month in 2016.

Considering the optimization algorithm and the cost coefficients, the BESS charges with the optimum rate to fill the valley while increasing the stored energy in the BESS. The energy of the BESS reaches to its highest point before the peak hours and then BESS starts to discharge with different rates with respect to the constraints. The optimization algorithm discharges the BESS at the points where it is beneficial. At the end of the daily cycle, the BESS charges back to the initial energy it started with. The charge/discharge operation of the BESS for the size of $280 \mathrm{kWh}$ is depicted in Fig. 5.



Fig. 5. BESS Energy changes for $280 \mathrm{kWh}$ capacity.

\section{B. BESS Sizing Optimization}

The BESS sizing algorithm gets the information from the cost minimization layer and uses the results to investigate different BESS capacities cost performance. The resulting capacity provides the highest benefit with respect to the investment cost of the BESS. The optimal size of the BESS is determined by investigating a range of capacities from $70 \mathrm{kWh}$ to $800 \mathrm{kWh}$. The total cost of different BESS capacities has been calculated and compared.

As specified in Fig. 6, comparing various capacities, the BESS with the capacity of $280 \mathrm{kWh}$ has the lowest total cost within a year. The total annual cost of $280 \mathrm{kWh}$ BESS is $\$ 695 \mathrm{k}$. Looking at the trend of changes in the BESS capacity graph, the optimization algorithm decreases the total cost as the BESS capacity increases until the capacity reaches its optimum capacity. Afterwards, by the rise in the capacity, the total annual cost grows with an approximately linear trend.



Fig. 6. BESS Optimal Capacity.

\section{Cost-benefit Analysis}

The cost-benefit analysis of different BESS capacities expresses the relationship between the time and the cost by employing PBP. Different BESS capacities from $70 \mathrm{kWh}$ to 800 $\mathrm{kWh}$ are considered for the analysis. Fig. 7 illustrates the PBP for different BESS capacities. According to the graph, a stepwise growth in the PBP is seen. In other words, the BESS capacities from 70 to $235 \mathrm{kWh}$ have the lowest PBP (4.33 years) and as the BESS size grows, the PBP increases in a linear stepwise trend. The PBP for the BESS capacity of $280 \mathrm{kWh}$ is 4.42 years as shown in the graph.



Fig. 7. PBP for BESS capacities from 70 to $800 \mathrm{kWh}$

\section{V.CONCLUSION}

The combination of RESs and BESS as a solution to lessen the costs of the MGs can moderate the dependence of the research center on the grid and increase the stability of the system. Investing on the BESS benefits the research center especially in the power outage cases. Besides shaving the peaks, 
the optimal scheduling of the BESS prevents power drops by filling the valleys. In addition, the BESS acts as backup power source in extreme cases such as power outage. The two-layer optimization algorithm proves the applicability of the BESS as a part of the MG to minimize the costs of the research center.

\section{REFERENCES}

[2] A. Saez-de-Ibarra, E. Martinez-Laserna, D. I. Stroe, M. Swierczynski, and P. Rodriguez, "Sizing Study of Second Life Liion Batteries for Enhancing Renewable Energy Grid Integration," IEEE Transactions on Industry Applications, vol. 52, no. 6, pp. 4999-5008, 2016.

[3] Y. Du, R. Jain, and S. M. Lukic, "A novel approach towards energy storage system sizing considering battery degradation," in 2016 IEEE Energy Conversion Congress and Exposition (ECCE), 2016, pp. 1-8.

[4] C. Mateo, J. Reneses, A. Rodriguez-Calvo, P. Frías, and S. Á, "Costbenefit analysis of battery storage in medium-voltage distribution networks," IET Generation, Transmission \& Distribution, vol. 10, no. 3, pp. 815-821, 2016.

[5] M. Dabbagh, A. Rayes, B. Hamdaoui, and M. Guizani, "Peak shaving through optimal energy storage control for data centers," in 2016 IEEE International Conference on Communications (ICC), 2016, pp. 1-6.

[6] A. Nagarajan and R. Ayyanar, "Design and Strategy for the Deployment of Energy Storage Systems in a Distribution Feeder With Penetration of Renewable Resources," IEEE Transactions on Sustainable Energy, vol. 6, no. 3, pp. 1085-1092, 2015.

[7] L. Dusonchet, M. G. Ippolito, E. Telaretti, G. Zizzo, and G. Graditi, "An optimal operating strategy for combined RES-based generators and electric storage systems for load shifting applications," in Power Engineering, Energy and Electrical Drives (POWERENG), 2013 Fourth International Conference on, 2013, pp. 552-557.

[8] J. Fedjaev, S. A. Amamra, and B. Francois, "Linear programming based optimization tool for day ahead energy management of a lithium-ion battery for an industrial microgrid," in 2016 IEEE International Power Electronics and Motion Control Conference (PEMC), 2016, pp. 406-411.

[9] B. Wang, M. Zarghami, and M. Vaziri, "Energy management and peak-shaving in grid-connected photovoltaic systems integrated with battery storage," in 2016 North American Power Symposium (NAPS), 2016, pp. 1-5.

[10] L. Bo and M. Shahidehpour, "Short-term scheduling of battery in a grid-connected PV/battery system," Power Systems, IEEE Transactions on, vol. 20, no. 2, pp. 1053-1061, 2005.

[11] A. Oudalov, R. Cherkaoui, and A. Beguin, "Sizing and Optimal Operation of Battery Energy Storage System for Peak Shaving Application," in Power Tech, 2007 IEEE Lausanne, 2007, pp. 621625.

[12] B. R. Ke, T. T. Ku, Y. L. Ke, C. Y. Chuang, and H. Z. Chen, "Sizing the Battery Energy Storage System on a University Campus With Prediction of Load and Photovoltaic Generation," IEEE Transactions on Industry Applications, vol. 52, no. 2, pp. 11361147, 2016.

[13] H. Dagdougui, N. Mary, A. Beraud-Sudreau, and L. Dessaint, "Power management strategy for sizing battery system for peak load limiting in a university campus," in 2016 IEEE Smart Energy Grid Engineering (SEGE), 2016, pp. 308-312.

[14] S. X. Chen, H. B. Gooi, and M. Q. Wang, "Sizing of Energy Storage for Microgrids," IEEE Transactions on Smart Grid, vol. 3, no. 1, pp. 142-151, 2012.

[15] Y. Yang, H. Li, A. Aichhorn, J. Zheng, and M. Greenleaf, "Sizing Strategy of Distributed Battery Storage System With High Penetration of Photovoltaic for Voltage Regulation and Peak Load Shaving," IEEE Transactions on Smart Grid, vol. 5, no. 2, pp. 982991, 2014.
[16] L. Xu, X. Ruan, C. Mao, B. Zhang, and Y. Luo, "An Improved Optimal Sizing Method for Wind-Solar-Battery Hybrid Power System," IEEE Transactions on Sustainable Energy, vol. 4, no. 3, pp. 774-785, 2013.

[17] M. Gitizadeh and H. Fakharzadegan, "Effects of electricity tariffs on optimal battery energy storage sizing in residential PV/storage systems," in Energy Efficient Technologies for Sustainability (ICEETS), 2013 International Conference on, 2013, pp. 1072-1077.

[18] T. A. Nguyen, M. L. Crow, and A. C. Elmore, "Optimal Sizing of a Vanadium Redox Battery System for Microgrid Systems," IEEE Transactions on Sustainable Energy, vol. 6, no. 3, pp. 729-737, 2015.

[19] A. K. Barnes, J. C. Balda, S. O. Geurin, and A. Escobar-Mejía, "Optimal battery chemistry, capacity selection, charge/discharge schedule, and lifetime of energy storage under time-of-use pricing," in 2011 2nd IEEE PES International Conference and Exhibition on Innovative Smart Grid Technologies, 2011, pp. 1-7.

[20] I. Alsaidan, A. Khodaei, and W. Gao, "Determination of optimal size and depth of discharge for battery energy storage in standalone microgrids," in 2016 North American Power Symposium (NAPS), 2016, pp. 1-6.

[21] R. Khare and Y. Kumar, "Application of GA on size optimization of solar-DG-battery storage HRES with reliability and $\mathrm{CO} 2$," in 2014 Annual IEEE India Conference (INDICON), 2014, pp. 1-7.

[22] Y. Yoon and Y.-H. Kim, "Charge Scheduling of an Energy Storage System under Time-of-Use Pricing and a Demand Charge," The Scientific World Journal, vol. 2014, p. 9, 2014, Art. no. 937329.

[23] M. O. Badawy, F. Cingoz, and Y. Sozer, "Battery storage sizing for a grid tied PV system based on operating cost minimization," in 2016 IEEE Energy Conversion Congress and Exposition (ECCE), 2016, pp. 1-7.

[24] T. Kerdphol, Y. Qudaih, and Y. Mitani, "Battery energy storage system size optimization in microgrid using particle swarm optimization," in IEEE PES Innovative Smart Grid Technologies, Europe, 2014, pp. 1-6.

[25] C. D. Rodnguez-Gallegos et al., "Placement and sizing optimization for PV-battery-diesel hybrid systems," in 2016 IEEE International Conference on Sustainable Energy Technologies (ICSET), 2016, pp. 83-89.

[26] H. Xiao, W. Pei, Y. Yang, and L. Kong, "Sizing of battery energy storage for micro-grid considering optimal operation management," in 2014 International Conference on Power System Technology, 2014, pp. 3162-3169.

[27] R. Garmabdari, M. Moghimi, and J. Lu, "Optimisation of Battery Energy Storage Capacity for a Grid-Tied Renewable Microgrid," presented at the 2017 IEEE Innovative Smart Grid Technologies Asia (ISGT-Asia), 2017.

[28] M. Moghimi, C. Bennett, D. Leskarac, S. Stegen, and L. Junwei, "Communication architecture and data acquisition for experimental MicroGrid installations," in Power and Energy Engineering Conference (APPEEC), 2015 IEEE PES Asia-Pacific, 2015, pp. 15 .

[29] B. Xu, A. Oudalov, A. Ulbig, G. Andersson, and D. Kirschen, "Modeling of Lithium-Ion Battery Degradation for Cell Life Assessment," IEEE Transactions on Smart Grid, vol. PP, no. 99, pp. $1-1,2016$.

[30] C. Zhou, K. Qian, M. Allan, and W. Zhou, "Modeling of the Cost of EV Battery Wear Due to V2G Application in Power Systems," IEEE Transactions on Energy Conversion, vol. 26, no. 4, pp. 1041-1050, 2011.

[31] C. Ju, P. Wang, L. Goel, and Y. Xu, "A Two-layer Energy Management System for Microgrids with Hybrid Energy Storage considering Degradation Costs," IEEE Transactions on Smart Grid, vol. PP, no. 99, pp. 1-1, 2017.

[32] T. Mesbahi, N. Rizoug, P. Bartholomeüs, R. Sadoun, F. Khenfri, and P. L. Moigne, "Optimal Energy Management for a Li-Ion Battery/Supercapacitor Hybrid Energy Storage System Based on a Particle Swarm Optimization Incorporating Nelder\&Mead Simplex Approach," IEEE Transactions on Intelligent Vehicles, vol. 2, no. 2, pp. 99-110, 2017. 